\title{
Research Article \\ Effect of Fiber Treatment and Fiber Loading on Mechanical Properties of Luffa-Resorcinol Composites
}

\author{
Chhatrapati Parida, ${ }^{1}$ Sarat Kumar Dash, ${ }^{2}$ and Sarat Chandra Das ${ }^{3}$ \\ ${ }^{1}$ Depatment of Physics, Orissa University of Agriculture and Technology, Bhubaneswar, Odisha 751 003, India \\ ${ }^{2}$ Department of Education in Science and Mathematics, National Council of Educational Research and Training, \\ Bhubaneswar, Odisha 751022, India \\ ${ }^{3}$ Department of Chemistry, Salipur College, Salipur, Odisha 754 202, India
}

Correspondence should be addressed to Chhatrapati Parida; sivaji_1976@yahoo.co.in

Received 8 January 2015; Accepted 31 January 2015

Academic Editor: Marino Lavorgna

Copyright (C) 2015 Chhatrapati Parida et al. This is an open access article distributed under the Creative Commons Attribution License, which permits unrestricted use, distribution, and reproduction in any medium, provided the original work is properly cited.

Tensile and compressive behaviour of resorcinol-formaldehyde (RF) matrix and its composites reinforced with fibers of Luffa cylindrica (LC) have been studied. LC fibers were subjected to chemical treatments such as alkali activation by $\mathrm{NaOH}$ followed by bleaching and acid hydrolysis in order to improve fiber-matrix adhesion. Both treated and untreated LC fibers are modified with calcium phosphate. The presence of hydroxy apatite, a polymorph of calcium phosphate and a major constituent of vertebrate bone and teeth, was confirmed from XRD peak of treated LC fiber. XRD analysis of the treated LC fiber has confirmed the crystalline nature of the chemically treated LC fiber by its crystallinity index. The effects of fiber loading of chemically treated and untreated LC fiber on ultimate stress, yield strength, breaking stress, and modulus of the composites were analyzed. The tensile and compressive modulus of the composites were increased with incorporation of both treated and untreated LC fibers into the RF matrix. The modulus of composites with treated LC fiber was enhanced compared to that of the untreated fiber composites. Furthermore the values of ultimate stress, yield stress, and breaking stress were increased with the incorporation of treated LC fiber in the composites.

\section{Introduction}

Plant biomass is composed of substances such as cellulose, lignin, and hemicellulose. Small amounts of pectin, pigments, and other extractives are also found in plant fiber [1]. However, cellulose is the major constituent of the vegetable organisms [2]. Cellulose is a linear syndiotactic homopolymer composed of D-anhydro-glucopyranose units (AGU) linked together by glycosidic bond. Each of AGU possesses hydroxy groups at three different positions. The size of chain is expressed by the degree of polymerization and average values of the degree of polymerization of the cellulose are in the range of 1000-15000 [3]. The Young modulus of cellulose is $137 \mathrm{GPa}$ [4] and is comparable to that of aramid fiber. Aramid fiber also known as kevlar is a synthetic fiber. Such high modulus of the cellulose microfibril is due to molecular alignment and close packing in its unit cell. The fibrillar model of cellulose suggests the relationships between fiber morphology and tensile properties. The tensile properties are related to the number of interlinks between the morphological units, degree of orientation, and degree of crystallinity of the molecules composing the fiber structure. The mechanical properties of cellulose based composites are utilized in construction of buildings, furniture, ropes, textiles, packaging industries, and so forth. The mechanical properties of natural fiber composites are much lower than those of glass fibers. However, their specific properties, especially stiffness, are comparable to the stated values of glass fibers [5]. Moreover, natural fibers are about 50\% lighter than glass and are in general cheaper. It is widely acknowledged that natural fiber composites combine good mechanical properties with a low specific mass and offer an alternative material to glass fiber-reinforced plastics in some technical applications. Thus, the study of mechanical properties of the composites using natural fiber appears to generate substantial interest. With this as an inspiration we expect the enhancement of 
mechanical properties of composites reinforced with Luffa cylindrica (LC) fiber which is a common tropical fruit of Orissa. Such fibers are composed of $60 \%$ cellulose, $30 \%$ hemicellulose, and $10 \%$ lignin [6]. The absence of a detailed study of its characteristics and properties in the scientific literature and high \% of cellulose makes this fiber material our topic of research.

The modern society is now facing a challenge to develop new materials for biomedical applications. Calcium phosphate based biomaterials are extensively used for bone replacement, dental filling, bone tissue engineering, drug delivery, and so forth [6]. The art of constructing complex inorganic structure over the surface of any material is known as biomimetic synthesis. However, biomimetic material will only be technologically relevant if developed through inexpensive route, with high product yield. Keeping this in mind we have attempted to explore the possibilities of using composites reinforced with LC fibers in biomedical industries by depositing compounds of calcium salts on the LC fibers. The bond strength of the natural fiber-reinforced composites is often lowered by the absorption of moisture. Natural fibers, being hydrophilic in nature, absorb moisture from the environment. This characteristic of the natural fibers leads to poor adhesion with the resin matrix, ultimately causing debonding between fiber and matrix. In wet conditions, therefore, such composites show very poor mechanical properties. Alkali treatment on natural fiber is a very common method usually used to remove hemicelluloses and lignin from them [7]. Alkali treatment increases hydrophobicity and leads to breaking down of the composite fiber bundle into smaller fibers. It also reduces fiber diameter and thereby increases the aspect ratio (width/height) which leads to the formation of a rough surface topography that results in better fiber-matrix interface adhesion [8]. Furthermore, this treatment also increases the number of possible reactive sites and allows better fiber wetting. In order to improve interfacial bonding and to reduce moisture absorption the LC fibers are treated with alkali.

Mechanical properties of natural fiber based composites have been studied by several researchers. Bledzki and Gassan in 1999 [1] reported that the characteristic mechanical properties of natural fibers are comparable to those of glass fibers. Wambua et al. in 2003 [9] while studying the effect of the volume fraction of fiber on the tensile strength of natural fiber composites found that the hemp fiber/polypropylene composites with a $30 \%$ volume fraction result in tensile strength of $52 \mathrm{MPa}$ which is higher than equivalent glassreinforced composites with the same volume fraction. Furthermore, hemp and kenaf fiber-polypropylene composites displayed tensile modulus of $6.8 \mathrm{GPa}$ as compared to $6.2 \mathrm{GPa}$ of equivalent glass composites. Nishino in 2004 [10] studied the mechanical properties of kenaf/poly-L-lactide (PLLA) composites and observed that the modulus of the composites increases with the increase of the volume fraction up to a threshold limit and then decreases with increase in volume fraction of fibers in the composites. Bos et al. in 2006 [11] reported that the modulus and the tensile strength increase with the increase of the volume or weight fraction on flax fiber/polypropylene composites when the fiber was treated with maleic anhydride. The preparation of nanocomposite films from microfibrillated cellulose (MFC) in combination with melamine formaldehyde was reported by Henriksson and Berglund in 2007 [12]. Cellulose films showed an average Young's modulus of $14 \mathrm{GPa}$ while in nanocomposites it was as high as $16.6 \mathrm{GPa}$ and average tensile strength as high as $142 \mathrm{MPa}$. Yan et al. in 2012 [13] while studying the properties of biocomposites using flax, linen, and bamboo fiber reinforced with epoxy using a vacuum bagging technique observed that the tensile and flexural strength of chemically treated flax/epoxy composite increased by $21.9 \%$ and $16.1 \%$ as compared to that of untreated composites. Siqueira et al. in 2010 [14] investigated the tensile modulus of LC nanocrystals and the maximum value of tensile modulus of LC nanocrystals was found to be $3.1 \mathrm{GPa}$.

\section{Experimental Measurements}

The LC fibers were cut into small pieces of length about $2 \mathrm{~cm}$, washed thoroughly with deionized water and then dried at $70^{\circ} \mathrm{C}$ in vacuum oven for 20 minutes. The dried LC fibers were treated with alkali followed by bleaching and acid hydrolysis. For giving alkali treatment, the LC fibers were soaked in $5 \% \mathrm{NaOH}$ solution at $80^{\circ} \mathrm{C}$ for $1 \mathrm{~h}$. During alkali treatment the hemicelluloses and lignin present in the LC fibers were extracted. In this way the number of $-\mathrm{OH}$ groups present in the fiber was reduced. The decrease in - $\mathrm{OH}$ groups with increase in hydrophobicity of natural fibers facilitated in strengthening the bonding between fiber and matrix. Such treatment also depolymerized the cellulose and exposed the short length crystallites of cellulose. The alkali treated LC fibers were bleached with $2 \%$ sodium hypochlorite solution under continuous stirring for $2 \mathrm{~h}$ at $80^{\circ} \mathrm{C}$. The colour of the bleached LC fibers changed from black to yellowish. Then the bleached LC fiber/water suspension was prepared and kept on an ice bath. $\mathrm{H}_{2} \mathrm{SO}_{4}$ was added slowly under continuous stirring to the suspension placed in an ice water bath, until the final concentration of $60 \% \mathrm{H}_{2} \mathrm{SO}_{4}$ was reached. The obtained suspension was heated at $45^{\circ} \mathrm{C}$ under continuous stirring for $2 \mathrm{~h}$. The mixture was washed and centrifuged using an ultracentrifuge at $30^{\circ} \mathrm{C}$ for 20 minutes with $7000 \mathrm{rpm}$ in order to remove excess acid.

The treated and untreated LC fiber were then immersed in $\mathrm{CaCl}_{2}$ solution for $12 \mathrm{~h}$ at room temperature of $28^{\circ} \mathrm{C}$ and washed with distilled water for removing excess calcium deposited on it. LC fibers modified with $\mathrm{CaCl}_{2}$ were reimmersed in $\mathrm{Na}_{2} \mathrm{HPO}_{4}$ solution for $12 \mathrm{~h}$ at room temperature to deposit compounds of calcium phosphate over it.

Composites are formed by taking resorcinol-formaldehyde (RF) resin and both chemically treated and chemically untreated LC fibers. The prepared grounded RF resin and LC fiber modified with calcium phosphate were mixed in different wt proportion which result in B1, B2, B3, B4, and B5 samples. In samples B1 and B2, the matrix and untreated LC fiber were in wt ratios $1: 1$ and $1: 2$, respectively. In samples B4 and B5, the matrix and treated LC fiber were in wt ratios $10: 1$ and $5: 1$, respectively with $B 3$ sample containing only powdered RF matrix. Maleic anhydride (1g) and ethylenediamine $(4.6 \mathrm{~mL})$ were added as cross-linkers to 
$1 \mathrm{~g}$ of $\mathrm{RF}$ resin. The mixture was heated at $70^{\circ} \mathrm{C}$ for $9 \mathrm{~h}$ to get composites. The grounded composite was then exposed to ultrasonic vibration at $30 \mathrm{kHz}$ for $1 \mathrm{~h}$ at room temperature of $28^{\circ} \mathrm{C}$ using a sonicator (1.5 L50 H/Deluxe, India) for dissolution of particles. $10 \mathrm{~g}$ of powdered composites was mixed with $10 \mathrm{~g}$ of commercial divinylbenzene and kept for $12 \mathrm{~h}$ at room temperature of $28^{\circ} \mathrm{C}$. The material was made acidic by addition of $0.25 \mathrm{~mL}$ of methanesulfonic acid. The sample was swollen as it absorbed sufficient divinylbenzene into it. This made the sample soft and a paste like material was obtained. The paste was kept in a small mould $(10 \mathrm{~cm} \times$ $2 \mathrm{~cm}$ ) and heated for $4 \mathrm{~h}$ at $60^{\circ} \mathrm{C}$ and then cured to get crosslinked composite.

The crystallinity index $\left(I_{c}\right)$ of the cellulose fibers was obtained from X-ray diffraction pattern using the following expression [15]:

$$
I_{c}(\%)=\frac{I-I_{\mathrm{am}}}{I} \times 100,
$$

where $I$ denotes the intensity of 002 plane and $I_{\text {am }}$ denotes the intensity of 101 plane of the amorphous phase. The measurement of tensile strength was undertaken in accordance with ASTM D 638 method and compressive strength was measured using ASTM D 682 method.

\section{Results and Discussion}

3.1. Crystallinity Index of Cellulose in Treated LC Fiber. Figure 1 shows the X-ray diffractogram of the treated fibers of LC modified with calcium phosphate in which three distinct peaks are observed at $15.87^{\circ}, 22.86^{\circ}$, and $34.64^{\circ}$. The peak at $15.87^{\circ}$ indicates amorphous cellulose or cellulose II of (101) crystallographic plane whereas the peak at $22.86^{\circ}$ indicates crystalline cellulose [cellulose I] of (002) crystallographic plane [15]. It shows that the treated LC fibers are partly crystalline and partly amorphous in nature. The unit cell of glucopyranose in cellulose molecule is monoclinic with two principal planes of reflection such as (002) and (101). The amount of crystalline cellulose or cellulose I in the total cellulose can be expressed by X-ray crystallinity index as defined by (1). The crystallinity index was found to be $60.3 \%$. The high crystallinity index of cellulose in treated LC fiber indicates the possibility of increasing the crystallinity of composites when the chemically treated LC fibers are reinforced into the matrix. However, the XRD peak at $34.64^{\circ}$ shows the presence of hydroxy apatite (JCPDS 34-0010) of (300) crystallographic plane in the treated LC fiber [16, 17]. Amorphous calcium phosphate comprises $80 \%$ volume of human bone [18]. Hydroxy apatite is the polymorph of calcium phosphate and is most extensively used in orthopedics, dentistry, and reconstructive surgery. It is biocompatible and bioinert. Characteristics such as release of calcium and phosphate ions during wound healing and defect filling are highly desirable. As such development of calcium phosphate based composites has drawn much attention in recent years and there are extensive efforts in the last few decades in developing calcium phosphate based composites [19].

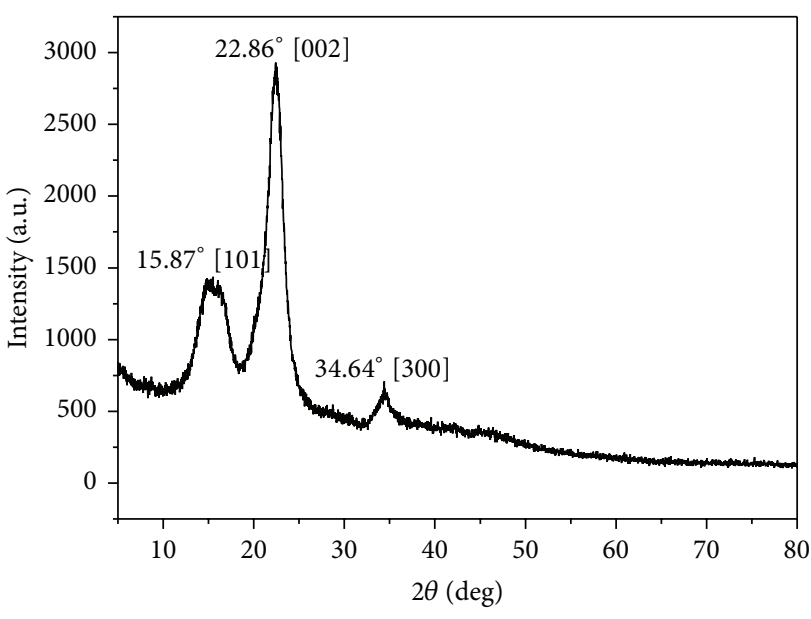

FIGURE 1: XRD spectra of treated fibers of LC modified by calcium phosphate.

Tables 1 and 2 summarize the tensile and compressive properties of matrix, composites with untreated and treated LC fibers.

3.2. Effect of Untreated Fiber Loading on Strength of Composites of LC Fiber. From Table 1 it is observed that the value of ultimate tensile stress of pure matrix (sample B3) is 15.744 MPa. But when the matrix is reinforced with untreated LC fiber in the wt ratio $1: 1$ the value of ultimate stress is decreased to $14.88 \mathrm{MPa}$ in sample B1. Again when the wt of untreated LC fiber in the matrix increases, that is, $2: 1$, the ultimate tensile stress is further decreased to $9.7 \mathrm{MPa}$ in sample B2. Such results are supported by the findings of ultimate compressive stress; that is, the ultimate compressive stress of $76.68 \mathrm{MPa}$ in matrix is decreased to $70 \mathrm{MPa}$ in sample $\mathrm{B} 1$ and $62.64 \mathrm{MPa}$ in sample B2. Thus the ultimate stress of composites with untreated LC fibers is lower as compared to pure matrix. The untreated LC fibers are highly hydrophilic in nature due to presence of cellulose, hemicellulose, and lignin and they have relatively higher affinity to absorb moisture which prevents adhesion between fiber and matrix. The decrease in strength of composites with untreated LC fibers in samples B1 and B2 may be due to poor adhesion between matrix and untreated LC fiber. The poor interfacial adhesion results in numerous voids at the interface between fiber and matrix. Due to presence of such voids the composites are inefficient for stress transfer.

3.3. Effect of Chemical Treatment of LC Fiber on Strength of Composites. When LC fibers are treated with alkali and bleached, there is enhancement of yield strength of composites with treated LC fibers as seen from Tables 1 and 2. Table 1 betokens that the value of ultimate tensile stress of matrix (sample B3) is $15.744 \mathrm{MPa}$. When the matrix is reinforced with treated LC fibers in wt ratios $10: 1$ (sample B4) and 5:1 (sample B5) the ultimate tensile stress is increased to $26.2 \mathrm{MPa}$ and $29.438 \mathrm{MPa}$, respectively. Therefore, tensile strength increased by $86.97 \%$ due to incorporation of treated 
TABLE 1: Values of ultimate tensile stress, yield strength, breaking stress, and tensile modulus of composites and matrix at different fiber loadings.

\begin{tabular}{lcccc}
\hline Sample & $\begin{array}{c}\text { Ultimate } \\
\text { tensile stress } \\
(\mathrm{MPa})\end{array}$ & $\begin{array}{c}\text { Yield } \\
\text { strength } \\
(\mathrm{MPa})\end{array}$ & $\begin{array}{c}\text { Breaking } \\
\text { stress }(\mathrm{MPa})\end{array}$ & $\begin{array}{c}\text { Tensile } \\
\text { modulus } \\
(\mathrm{MPa})\end{array}$ \\
\hline B1 & 14.88 & 12.7 & 13.5 & 680 \\
B2 & 9.7 & 7.59 & 9 & 387 \\
B3 & 15.744 & 13.2 & 14 & 219 \\
B4 & 26.2 & 23.8 & 24.6 & 1440 \\
B5 & 29.438 & 27 & 28.2 & 1662 \\
\hline
\end{tabular}

TABLE 2: Values of ultimate compressive stress, yield strength, breaking stress, and compressive modulus of composites and matrix at different fiber loadings.

\begin{tabular}{lcccc}
\hline Sample & $\begin{array}{c}\text { Ultimate } \\
\text { Compres- } \\
\text { sive stress } \\
(\mathrm{MPa})\end{array}$ & $\begin{array}{c}\text { Yield } \\
\text { strength } \\
(\mathrm{MPa})\end{array}$ & $\begin{array}{c}\text { Breaking } \\
\text { stress (MPa) }\end{array}$ & $\begin{array}{c}\text { Compressive } \\
\text { modulus } \\
(\mathrm{MPa})\end{array}$ \\
\hline B1 & 70.28 & 59.5 & 66 & 2687 \\
B2 & 62.64 & 52 & 58.8 & 1483 \\
B3 & 76.68 & 66.51 & 73 & 1442 \\
B4 & 79.39 & 68.24 & 75.1 & 3440 \\
B5 & 81.00 & 70.24 & 77 & 4479 \\
\hline
\end{tabular}

LC fiber in the composites. Similarly $6 \%$ enhancement in compressive strength is also observed when treated LC fibers are used as reinforcement in the composites as compared to that of the matrix. However, due to incorporation of untreated LC fiber into the matrix, a drop of $5.4 \%$ in tensile strength and $9.2 \%$ in compressive strength were observed. Figure 2 shows the variation of ultimate tensile stress with different fiber loading and with chemical treatment on LC fibers.

The chemical treatment, namely, alkali treatment, followed by bleaching and acid hydrolysis is attempted in order to improve hydrophobic nature of LC fiber. When LC fibers are subjected to chemical treatment, wax, fatty acids, hemicellulose, and lignin are removed. Removal of these substances results in a rough surface with pits and that may lead to an increase in mechanical interlocking between treated LC fiber and matrix. The treatment probably exposes higher amount of cellulose on the fiber surface. Higher amount of cellulose reveals more reaction sites which again lead to better adhesion between treated LC fiber and matrix. Finally the chemical treatment on LC fiber causes fibrillation of fiber to small fibrils. With the decrease in fiber diameter, surface area increases which leads to more wet ability of fibers within the matrix. The high interfacial area is the most favorable aspect of composites with treated fibers [20].

The values of ultimate stress, yield stress, and breaking stress (Tables 1 and 2) are thus indicative of fiber-matrix adhesion in composites. The more the interfacial adhesion between fiber and matrix, the more the values of tensile and compressive strength. When the wt of LC fiber in the matrix

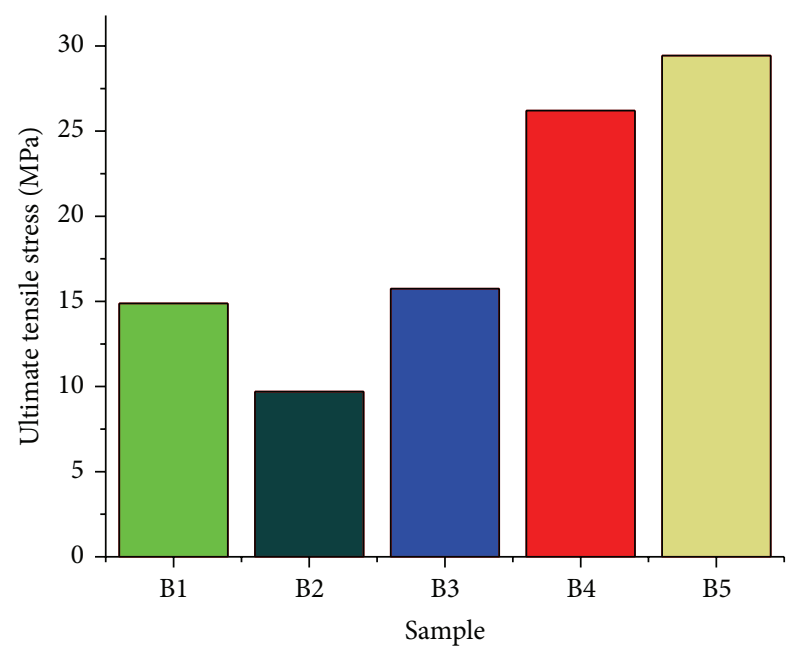

FIGURE 2: Variation of ultimate tensile stress with fiber loading for B1, B2, B3, B4, and B5 samples.

is decreased as in treated LC fiber composites in sample B4 (treated fiber: matrix $=1: 10$ ) and sample B5 (treated fiber: matrix $=1: 5$ ), the tensile strength of composites are increased by $87 \%$ as observed in Table 1 . The results indicate that with very low wt of treated LC fiber in the composites, the strength of the composites were nearly doubled in comparison to the strength of untreated LC fiber composites. Thus the specimens B4 and B5 are light wt materials with improved mechanical strength. When LC fibers are subjected to alkali treatment and bleaching, the size of fibrils decreases up to $100 \mathrm{~nm}$. With fibrillation of LC fiber due to chemical treatment, effective surface area of fiber increases. Due to large increase in surface area probably, dispersion of fiber was possible in the matrix with very low content of treated LC fiber as in sample B4 and sample B5. It leads to dramatic improvement in mechanical properties of sample B4 and sample B5.

3.4. Effect of Fiber Loading and Fiber Treatment on Modulus of Composites. The rigidity of natural fibers is more than the pure matrix which leads to an increase in modulus with the incorporation of fibers. From Table 1 it is observed that the value of tensile modulus of the matrix (sample B3) is $219 \mathrm{MPa}$. When the composites are formed with untreated LC fibers the tensile modulus is increased to maximum value of $680 \mathrm{MPa}$ in sample B1. Furthermore, in case of treated LC fiber composite the value of tensile modulus is increased to $1662 \mathrm{MPa}$ in sample B5. From Table 2 it is also observed that compressive modulus of $1442 \mathrm{MPa}$ in matrix was enhanced to a maximum value of $2687 \mathrm{MPa}$ in sample $\mathrm{B} 1$ and $4479 \mathrm{MPa}$ in sample B5. Thus incorporation of treated and untreated LC fiber increases the stiffness of the composites. However, the ductile behavior of all the composites is reduced due to incorporation of rigid fibers. Similar decreasing trends are observed for results in compressive modulus. The decrease of modulus with increase in wt of untreated LC fiber in the composites may be due to low dispersion of fibers in the matrix. On the 


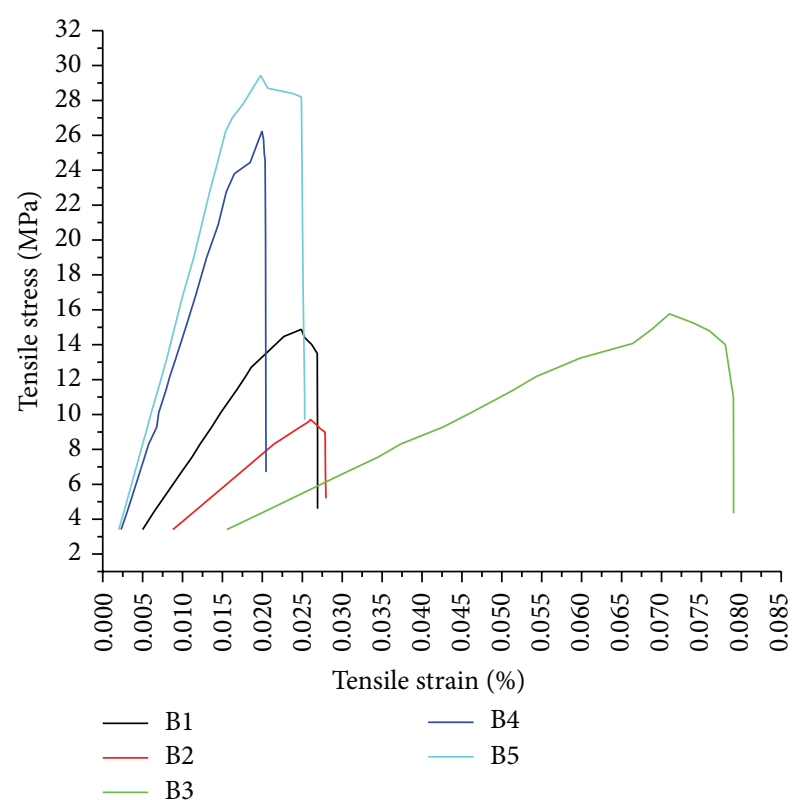

FIgURE 3: Tensile stress versus strain curve for B1, B2, B3, B4, B5 samples.

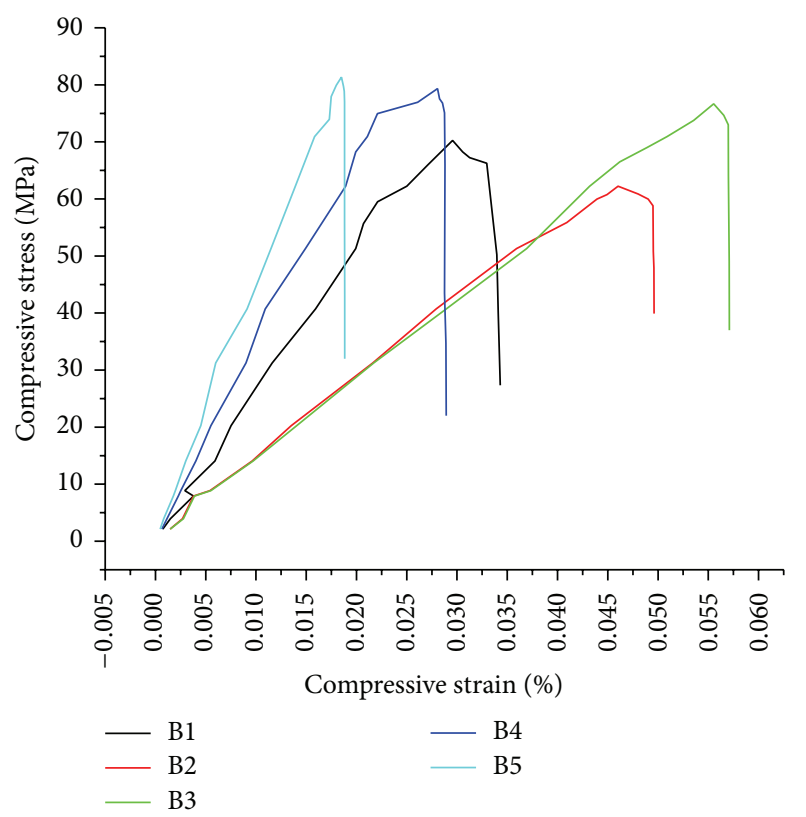

FIGURE 4: Compressive stress versus strain curve for B1, B2, B3, B4, and B5 samples.

other hand there is an increase in modulus when the wt of treated fibers increases indicating enhancement in stiffness with incorporation of more wt of treated LC fiber in the composites.

The stress versus strain curve for all the samples is shown in Figures 3 and 4 where Figure 3 represents tensile stress versus strain curve and Figure 4 represents compressive stress versus strain curve.
From Figures 3 and 4 it is observed that with the increase in wt of the LC fiber in the composite the stress versus strain curve shifts towards lower values of percentile compressive strain region which indicates an increase in modulus of the composites. However, shifting is appreciably more in case of treated LC fiber composites which indicates enhancement of modulus or stiffness due to incorporation of treated LC fibers.

\section{Conclusion}

The maximum values of tensile modulus and the compressive modulus of LC fiber, resorcinol formaldehyde composites, are found to be $1.662 \mathrm{GPa}$ and $4.479 \mathrm{GPa}$, respectively. The maximum value of tensile modulus $820 \mathrm{MPa}$ in LC fiber reinforced unsaturated polyester composites reported by Paglicawan in 2005 [21]. However, in our studies incorporation of both treated and untreated LC fibers into the matrix enhances the stiffness of the composites. Stiffness of the treated fiber composites is observed to be higher than stiffness of untreated fiber composites. Tensile strength and compressive strength of the composites decreased with incorporation of untreated fibers but strength of composites increases with incorporation of treated LC fibers. Thus there is an improvement observed in strength and stiffness of the composites for very low content of treated LC fiber in the composites as compared to that of the untreated LC fiber composites leading to light wt materials having higher strength to mass ratio. Such increase in mechanical strength of composites with incorporation of treated LC fiber is an indication of enhanced chemical bonding between treated LC fiber and matrix and it is supported by results from XRD analysis of treated LC fibers. The composites using LC fibers having mechanical strength as mentioned above may be suitable for use as low strength materials such as house panels, doors, windows, and fiber board [22].

\section{Conflict of Interests}

The authors declare that there is no conflict of interests regarding the publication of this paper.

\section{References}

[1] A. K. Bledzki and J. Gassan, "Composites reinforced with cellulose based fibres," Progress in Polymer Science, vol. 24, no. 2, pp. 221-274, 1999.

[2] M. S. Sreekala, M. G. Kumaran, and S. Thomas, "Green composites from natural rubber and oil," Journal of Applied Polymer Science, vol. 66, no. 5, pp. 821-835, 1997.

[3] R. J. Moon, A. Martini, J. Nairn, J. Simonsen, and J. Youngblood, "Cellulose nanomaterials review: structure, properties and nanocomposites," Chemical Society Reviews, vol. 40, no. 7, pp. 3941-3994, 2011.

[4] I. Sakurada, Y. Nukushina, and T. Ito, "Experimental determination of the elastic modulus of crystalline regions in oriented polymers," Journal of Polymer Science, vol. 57, no. 165, pp. 651660, 1962.

[5] J. G. Yu, M. Lei, and B. Cheng, "Morphology control of $\mathrm{SrCO}_{3}$ crystals using complexons as modifiers in the ethanol-water 
mixtures," Journal of Solid State Chemistry, vol. 177, pp. 681-689, 2004.

[6] I. O. Mazali and O. L. Alves, "Morphosynthesis: high fidelity inorganic replica of the fibrous network of loofa sponge ( Luffa cylindrical)," Anais da Academia Brasileira de Ciências, vol. 77, no. 1, pp. 25-31, 2005.

[7] D. Ray, B. K. Sarkar, A. K. Rana, and N. R. Bose, "Effect of alkali treated jute fibres on composite properties," Bulletin of Materials Science, vol. 24, no. 2, pp. 129-135, 2001.

[8] K. Joseph, L. H. C. Mattoso, R. D. Toledo et al., "Industrial fibres: recent and current developments," Polymer and Agrofiber, vol. 4, pp. 159-201, 2000.

[9] P. Wambua, J. Ivens, and I. Verpoest, "Natural fibres: can they replace glass in fibre reinforced plastics?" Composites Science and Technology, vol. 63, no. 9, pp. 1259-1264, 2003.

[10] T. Nishino, "Natural fiber sources," in Green Composites: Polymer Composites and the Environment, CRC Press, Boca Raton, Fla, USA, 2004.

[11] H. L. Bos, J. Müssig, and M. J. A. van den Oever, "Mechanical properties of short-flax-fibre reinforced compounds," Composites Part A: Applied Science and Manufacturing, vol. 37, no. 10, pp. 1591-1604, 2006.

[12] M. Henriksson and L. A. Berglund, "Structure and properties of cellulose nanocomposite films containing melamine formaldehyde," Journal of Applied Polymer Science, vol. 106, no. 4, pp. 2817-2824, 2007.

[13] L. Yan, N. Chouw, and X. Yuan, "Improving the mechanical properties of natural fibre fabric reinforced epoxy composites by alkali treatment," Journal of Reinforced Plastics and Composites, vol. 31, no. 6, pp. 425-437, 2012.

[14] G. Siqueira, J. Bras, and A. Dufresne, "Luffa as a cellulose source," BioResources, vol. 5, no. 2, pp. 727-740, 2010.

[15] J. Wang, Y. Zheng, and A. Wang, "Effect of kapok fiber treated with various solvents on oil absorbency," Industrial Crops and Products, vol. 40, no. 1, pp. 178-184, 2012.

[16] D. K. Pattanayak, P. Divya, S. Upadhyay, R. C. Prasad, B. T. Rao, and T. R. Rama Mohan, "Synthesis and evaluation of hydroxyapatite ceramics," Trends in Biomaterials and Artificial Organs, vol. 18, no. 2, pp. 169-175, 2005.

[17] JCPDS File No. 34-0010 (HAp), International Center for Diffraction Data.

[18] P. S. Prevéy, "X-ray diffraction characterization of crystallinity and phase composition in plasma-sprayed hydroxyapatite coatings," Journal of Thermal Spray Technology, vol. 9, no. 3, pp. 369$376,2000$.

[19] E. Caroline, E. Victoria, and F. D. Gnanam, "Synthesis and characterisation of biphasic calcium phosphate," Trends in Biomaterials \& Artificial Organs, vol. 16, no. 1, pp. 12-14, 2002.

[20] G. Goud and R. N. Rao, "Effect of fibre content and alkali treatment on mechanical properties of Roystonea regia-reinforced epoxy partially biodegradable composites," Bulletin of Materials Science, vol. 34, no. 7, pp. 1575-1581, 2011.

[21] M. A. Paglicawan, "Loofah fiber as reinforcement material for composite," Philippine Journal of Science, vol. 134, no. 2, pp. 113120, 2005.

[22] M. A. Dweib, B. Hu, H. W. Shenton III, and R. P. Wool, "Biobased composite roof structure: manufacturing and processing issues," Composite Structures, vol. 74, no. 4, pp. 379-388, 2006. 

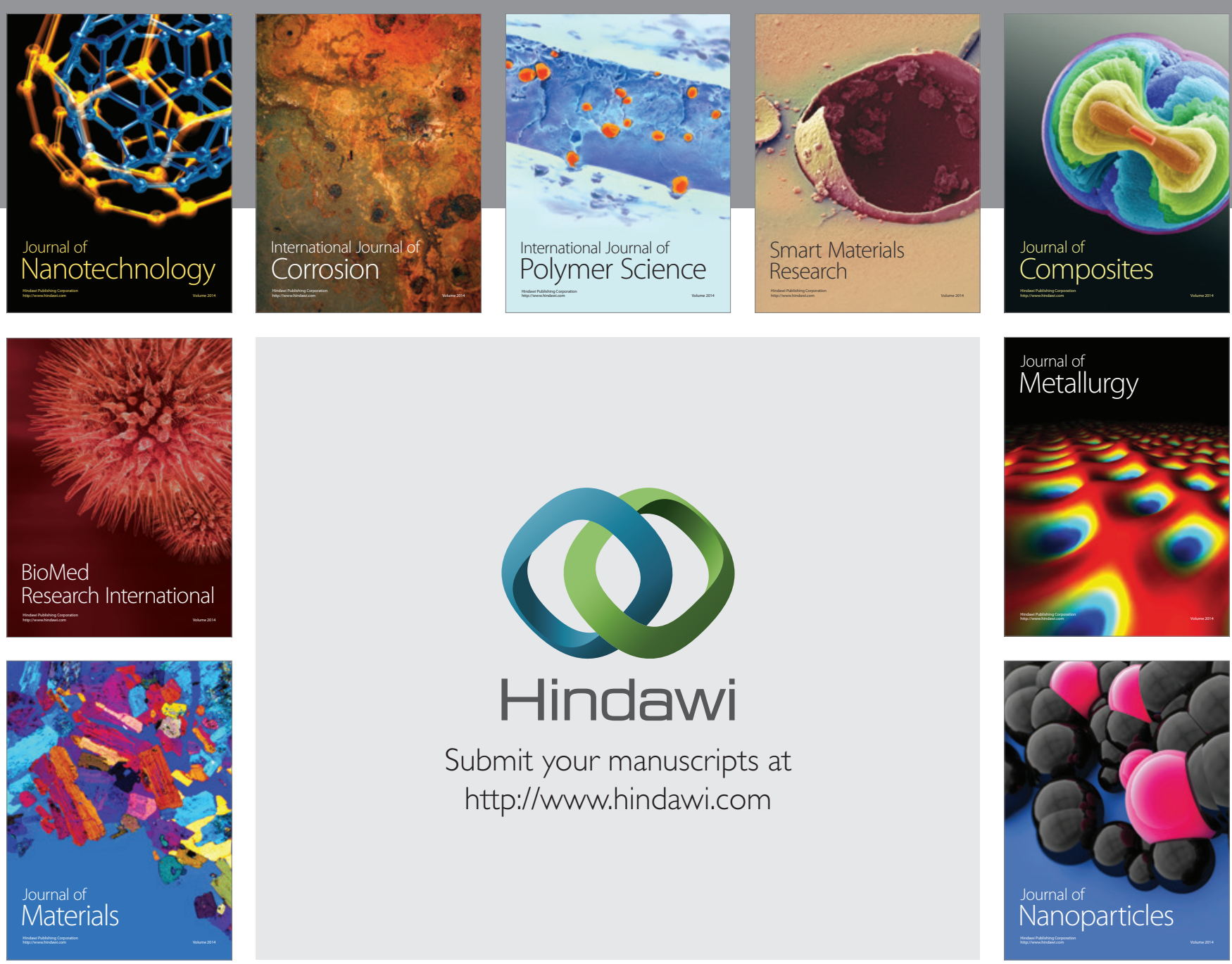

Submit your manuscripts at http://www.hindawi.com
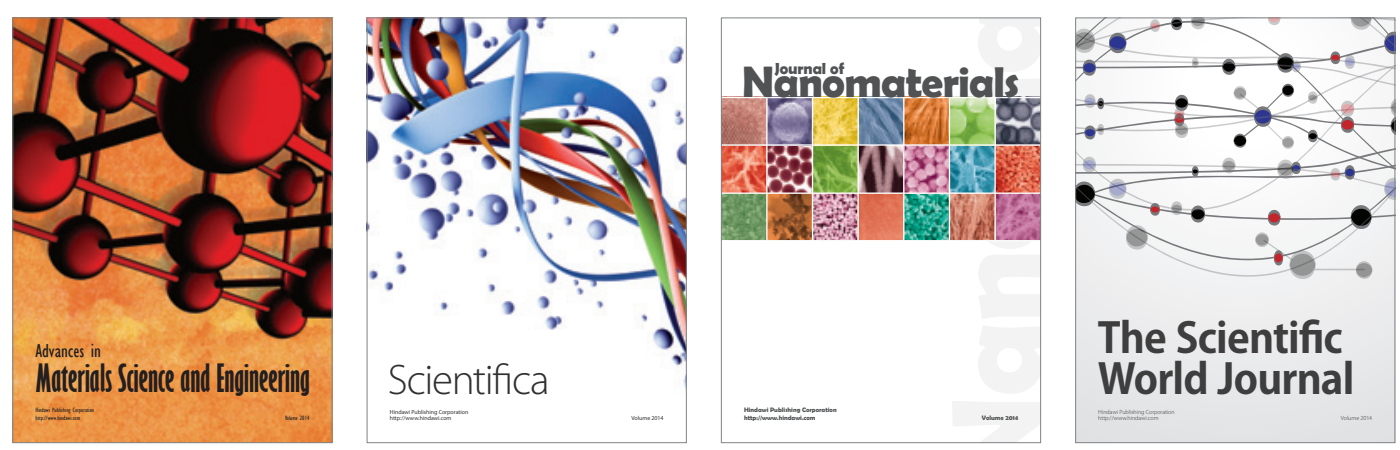

\section{The Scientific World Journal}
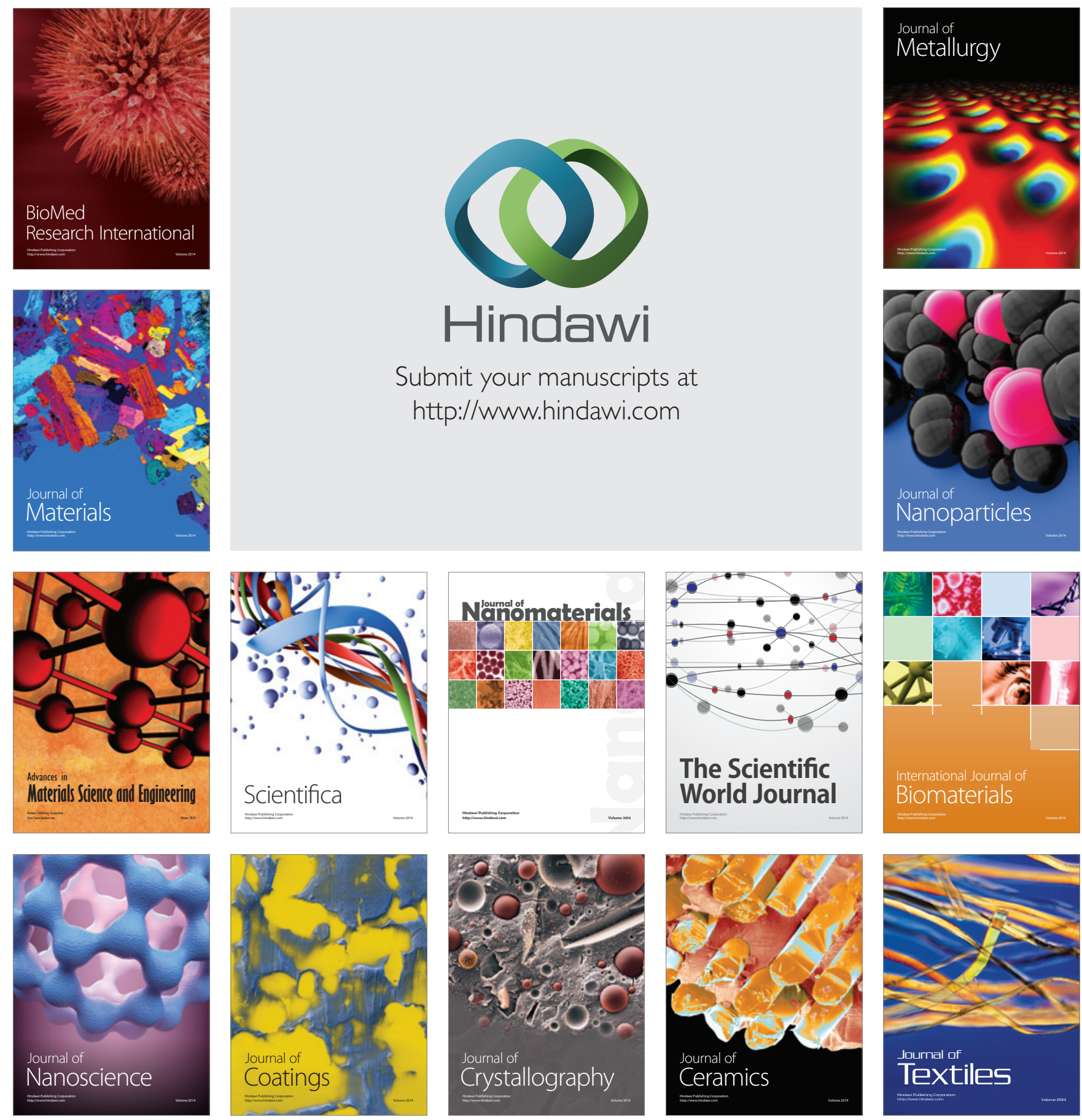